\title{
Black hole evaporation and semiclassicality at large $D$
}

\author{
Frederik Holdt-Sørensen $\odot,{ }^{1,2, *}$ David A. McGady, ${ }^{1,2,3,}$ and Nico Wintergerst ${ }^{1, \hbar}$ \\ ${ }^{1}$ Niels Bohr Institute, Blegdamsvej 17, DK-2100 Copenhagen $\varnothing$, Denmark \\ ${ }^{2}$ Niels Bohr International Academy, Blegdamsvej 17, DK-2100 Copenhagen $\emptyset$, Denmark \\ ${ }^{3}$ Nordita, KTH Royal Institute of Technology and Stockholm University, \\ Roslagstullsbacken 23, SE-106 91 Stockholm, Sweden
}

(Received 29 August 2019; accepted 16 June 2020; published 15 July 2020)

\begin{abstract}
Black holes of sufficiently large initial radius are expected to be well described by a semiclassical analysis at least until half of their initial mass has evaporated away. For a small number of spacetime dimensions, this holds as long as the black hole is parametrically larger than the Planck length. In that case, curvatures are small, and backreaction onto geometry is expected to be well described by a time-dependent classical metric. We point out that at large $D$, small curvature is insufficient to guarantee a valid semiclassical description of black holes. Instead, the strongest bounds come from demanding that the rate of change of the geometry is small and that black holes scramble information faster than they evaporate. This is a consequence of the enormous power of Hawking radiation in $D$ dimensions due to the large available phase space and the resulting minuscule evaporation times. Asymptotically, only black holes with entropies $S \geq D^{D+3} \log D$ are semiclassical. We comment on implications for realistic quantum gravity models in $D \leq 26$ as well as relations to bounds on theories with a large number of gravitationally interacting light species.
\end{abstract}

DOI: $10.1103 /$ PhysRevD.102.026016

\section{INTRODUCTION}

Generic (nonextremal) black holes famously have both finite entropies and temperatures, which together lead to Hawking radiance/luminosity and, eventually, complete evaporation. Absent a full treatment within quantum gravity, one studies evaporation within the semiclassical approximation, where the length scale given by Newton's constant, $\ell_{\mathrm{P}}:=G_{N}^{1 /(D-2)}=M_{\mathrm{P}}^{-1}=t_{\mathrm{P}}$, vanishes compared to the length scales of the geometry. Geometric backreaction via quantum mechanical fluctuations then can be safely ignored, which lets the radiation be cleanly computed. A posteriori, one then assumes that the flux calculated in the semiclassical approximation is accurate even for a finite mass black hole.

The validity of this scheme has been the subject of active debate for several decades. It necessarily breaks down at the very late stages of black hole evaporation, when curvatures become large compared to the Planck scale, requiring a full

\footnotetext{
*ndx115@alumni.ku.dk

dmcgady@alumni.princeton.edu

"nwintergerst@gmail.com
}

Published by the American Physical Society under the terms of the Creative Commons Attribution 4.0 International license. Further distribution of this work must maintain attribution to the author(s) and the published article's title, journal citation, and DOI. Funded by SCOAP. treatment in quantum gravity. On the other hand, resolution of the black hole information paradox-usually phrased as the tendency of semiclassical black holes to turn pure quantum states into mixed ones [1], a phenomenon that would be in clear tension with basic postulates of quantum mechanics-suggests a breakdown at much earlier stages. There is compelling evidence that this should happen at the so-called Page time [2] by which an initially classical black hole has lost roughly half its initial area via evaporation.

In this paper, we do not further explore the issue of information loss, even though we hope that our findings might provide nontrivial insights. Instead, we focus on the much more innocent question about the properties that allow for a semiclassical treatment of the early stages of black hole evaporation. In four spacetime dimensions, this is well understood. Whenever the Schwarzschild radius is large compared to the Planck scale, a black hole is semiclassical at least up to its Page time [3]. We show that when the spacetime dimension $D$ is sufficiently large, this ceases to be true for a class of "large" black holes.

Recent studies of the limit of a large number of spacetime dimensions $D$ have led to a better understanding of the aspects of classical general relativity, especially for black holes [4,5]. Yet, semiclassical (and fully quantum) features of black holes [6-9] within these new formulations of this large- $D$ limit seem to be relatively unexplored. This paper is yet another partial step in this direction. 
The main thrust of our work stems from the evaporation and scrambling timescales of Schwarzschild black holes in $D$ dimensions. The scrambling time is the time that a black hole needs to process and obscure infalling information [10]. It has been conjectured to saturate various bounds, bounds which are necessary for an evaporating black hole not to violate basic properties of quantum mechanics $[10,11]$. At large $D$, black holes coupled to $N_{D}$ massless modes, with Bekenstein-Hawking entropy $S_{\mathrm{BH}}$ and Hawking temperature $T_{\mathrm{H}}$, evaporate and scramble on the timescales:

$$
\begin{gathered}
\frac{t_{\text {evap }}}{t_{\mathrm{P}}} \sim\left(\frac{4 \pi}{D}\right)^{D+1 / 2} \frac{S_{\mathrm{BH}}}{N_{D}} \times S_{\mathrm{BH}}^{\frac{1}{D-2}}, \\
\frac{t_{\mathrm{scr}}}{t_{\mathrm{P}}}=\frac{M_{\mathrm{P}}}{2 \pi T_{\mathrm{H}}} \log S_{\mathrm{BH}} \sim S_{\mathrm{BH}}^{\frac{1}{D-2}} \times \frac{\log S_{\mathrm{BH}}}{D^{1 / 2}},
\end{gathered}
$$

where we have assumed the usual expression [10,11] for the scrambling time to hold in general $D$, a premise that we will discuss more towards the end of the paper. We have parametrized the timescales in terms of the entropy $S_{\mathrm{BH}}$ as it is the only dimensionless quantity in pure gravity in asymptotically flat spacetimes. As such, it allows us to capture the entire $D$ scaling without having to consider the individual $D$ dependence of dimensionful quantities, such as Newton's constant or the Schwarzschild radius.

Even from a very superficial view on black hole information processing, it seems clear that a black hole cannot evaporate faster than it scrambles information. Any black hole that appears to do so cannot possibly evaporate thermally and thus be described by semiclassical physics. Yet, for fixed $S_{\mathrm{BH}}$, the ratio of the scrambling and evaporation times indeed increases as $t_{\text {scr }} / t_{\text {evap }} \sim$ $D^{D} N_{D} \log S_{\mathrm{BH}} / S_{\mathrm{BH}}$. Thus, any fixed entropy black hole can be described by semiclassical physics only up to some critical dimension $D_{\text {crit }}\left(S_{\mathrm{BH}}\right)$. Properly semiclassical black holes have bounded entropy:

$$
S_{\mathrm{BH}} \gtrsim D^{D+3} \log D .
$$

This implies that semiclassical black holes have $\frac{R_{\mathrm{H}}}{\ell_{\mathrm{P}}} \gtrsim D^{3 / 2}$. We stress that we work with manifestly dimensionless ratios and thus may safely ignore how $\ell_{\mathrm{P}}=t_{\mathrm{P}}=1 / M_{\mathrm{P}}$ scale with the spacetime dimension $D$.

\section{SCHWARZSCHILD IN $D$ DIMENSIONS}

In this section, we first give general properties of $D$-dimensional Schwarzschild black holes. We then find their semiclassical evaporation and scrambling times.

\section{A. Metric, entropy, and temperature}

The metric for the $D$-dimensional Schwarzschild black hole with ADM mass $M_{\mathrm{H}}$ is well known to be

$$
\begin{gathered}
d s^{2}=-h_{D}(r) d t^{2}+\frac{d r^{2}}{h_{D}(r)}+r^{2} d \Omega_{D-2}, \\
h_{D}(r)=1-\left(\frac{R_{\mathrm{H}}}{r}\right)^{D-3}, \quad\left(\frac{R_{\mathrm{H}}}{\ell_{\mathrm{P}}}\right)^{D-3}=\frac{M_{\mathrm{H}}}{M_{\mathrm{P}}} \frac{16 \pi / \Omega_{D-2}}{(D-2)},
\end{gathered}
$$

where $\Omega_{D-2} \equiv 2 \pi^{\frac{D-1}{2}} / \Gamma\left(\frac{D-1}{2}\right)$ is the area of the $(D-2)$ dimensional unit sphere. The periodicity properties of $g_{00}(r)=-h_{D}(r)$ in the Euclidean signature directly give the Hawking temperature:

$$
t_{E} \sim t_{E}+\beta_{\mathrm{BH}}, \quad T_{\mathrm{H}}=\frac{1}{\beta_{\mathrm{BH}}}=\frac{(D-3)}{4 \pi R_{\mathrm{H}}} .
$$

This factor of $D$ is responsible for the high luminosities that are the focus of this paper. It is straightforward to see that the semiclassical Bekenstein-Hawking entropy is

$$
S_{\mathrm{BH}}:=\frac{A_{\mathrm{BH}}}{4 G_{N}}=\frac{\Omega_{D-2}}{4}\left(\frac{R_{\mathrm{H}}}{\ell_{\mathrm{P}}}\right)^{D-2}=\frac{4 \pi}{D-2} R_{\mathrm{H}} M_{\mathrm{H}} .
$$

The black hole's area is $A_{\mathrm{BH}}$; again $G_{N}:=\ell_{\mathrm{P}}^{D-2}$. We frame our main discussion in terms of the entropy $S_{\mathrm{BH}}$.

\section{B. Evaporation times and scrambling times}

We now combine properties of $D$-dimensional black holes with those of blackbodies in $D$ dimensions. Straightforward computations, for example in [12], give the following luminosity of a spherical blackbody with radius $R$ and temperature $T$ in $D$ dimensions:

$$
P_{D}=\left(N_{D} D\right) \frac{(T R)^{D}}{R^{2}} \frac{D-1}{D} \frac{\zeta(D)}{\pi} .
$$

Apart from dimensionless factors of $D$, this expression is simple to understand. Since the power of blackbodies is proportional to their area, one has $P_{D} \sim R^{D-2}$. Dimensional analysis then fixes the scaling with $T$. Finally, summing over all decay channels gives a factor of $N_{D}$. Note that $\frac{D-1}{D} \zeta(D)$ rapidly goes to 1 at large $D$.

We now use the properties of black holes as approximate blackbody radiators in $D$ dimensions. The $D$-dimensional Bose-Einstein distribution for temperature $T=T_{\mathrm{H}}$ peaks near

$$
E \sim E^{\star}:=(D-1) T_{\mathrm{H}}=(D-1) \frac{D-3}{4 \pi R_{\mathrm{H}}} \simeq \frac{D^{2}}{4 \pi R_{\mathrm{H}}} .
$$

Thus, when $D \gg 1$, black hole radiance is dominated by wavelengths that are small compared to $R_{\mathrm{H}}$. This excises greybody physics - which we parametrize and denote by the factor $\gamma_{D}\left(R_{\mathrm{H}}\right)$-from contributing to black hole radiance for even moderate values of $D$ (e.g., $D \gtrsim 8$ ) [6]. 
There is a further, slight, modification of the absorption/ emission area of the black hole. Rather than being a function of $R_{\mathrm{H}}$, it is parametrized by the maximum critical impact parameter $b_{\mathrm{C}}$, below which null rays are captured by a black hole [12]: $\frac{b_{\mathrm{C}}}{R_{\mathrm{H}}}=\left(\frac{D-1}{2}\right)^{1 /(D-3)}\left(\frac{D-1}{D-3}\right)^{1 / 2}$.

Combining these yields the black hole luminosity

$P_{\mathrm{BH}}=-\frac{d M_{\mathrm{H}}}{d t}=\frac{8 \pi}{e^{2}} \frac{N_{D}}{R_{\mathrm{H}}^{2}}\left(\frac{D}{4 \pi}\right)^{D+2} \times K_{D} \times \gamma_{D}\left(R_{\mathrm{H}}\right)$,

where both $K_{D}$ [defined in the Appendix in Eq. (A1)] and $\gamma_{D}\left(R_{\mathrm{H}}\right)$ (which is a proxy for greybody physics contributing to the luminosity) approach unity as $D$ grows [6]. Note that the most important factor in $P_{\mathrm{BH}},(D / 4 \pi)^{D}$, fundamentally comes from the fact that $R_{\mathrm{H}} T_{\mathrm{H}}=\frac{D-3}{4 \pi}$.

Thus, a black hole with entropy $S_{\mathrm{BH}}$ evaporates after

$$
\frac{t_{\text {evap }}}{t_{\mathrm{P}}}=\frac{e^{2}}{8 \pi} \sqrt{\frac{2}{e}}\left(\frac{D}{4 \pi}\right)^{-\left(D+\frac{1}{2}\right)} \frac{S_{\mathrm{BH}}}{N_{D}} \times S_{\mathrm{BH}}^{\frac{1}{D-2}} \times \frac{L_{D}}{K_{D}},
$$

where $L_{D}$, defined in Eq. (A2), also goes to unity for increasing $D$. This expression for $t_{\text {evap }}$ comes from rewriting $M_{\mathrm{H}}$ in terms of $S_{\mathrm{BH}}$. We recover Eq. (1) as $K_{D} / L_{D} \rightarrow 1$ and $(D-3)^{2} /(D(D-1)) \rightarrow 1$.

Finally, the scrambling time is [11]

$$
\frac{t_{\mathrm{scr}}}{t_{\mathrm{P}}}:=\frac{M_{\mathrm{P}}}{2 \pi T_{\mathrm{H}}} \log S_{\mathrm{BH}}=2 \frac{\log S_{\mathrm{BH}}}{D-3} \times\left(\frac{4 S_{\mathrm{BH}}}{\Omega_{D-2}}\right)^{\frac{1}{D-2}} .
$$

As is well known, in $D=4$ a large black hole, with $S_{\mathrm{BH}} \approx\left(R_{\mathrm{H}} / \ell_{\mathrm{P}}\right)^{2} \gg 1$, will scramble significantly faster than it evaporates: $t_{\text {scr }} \ll t_{\text {evap }}$. However, the factor of $S_{\mathrm{BH}} /\left(\frac{D}{4 \pi}\right)^{D}$ in $t_{\text {evap }}$ makes room for large black holes in $D \gg 1$ that have both $S_{\mathrm{BH}} \gg 1$ and $t_{\text {scr }} \gg t_{\text {evap }}$.

\section{SEMICLASSICAL PHYSICS AND LARGE $D$}

Towards the end of the evaporation process, the semiclassical analysis is expected to break down for any black hole. For instance, we expect that once the evaporation has proceeded to a sufficiently advanced point, the dynamics of the black hole is no longer well described by the background spacetime evolving solely according to the classical Einstein equations. Nonetheless, for sufficiently large initial entropies, the evaporation and scrambling times are very well approximated by the expressions in Eqs. (11) and (12). In this section, we make this statement more precise. In particular, we identify sources of the breakdown of semiclassicality, such as large curvature at the horizon or quasistaticity of the geometry. We will see that at sufficiently large $D$, the strongest bound is obtained from demanding scrambling times to be short as compared to the black hole lifetime.
To this end, it is useful to discuss black hole families in $D$ dimensions, indexed by positive numbers $k$ and $\hat{S}_{0}$ :

$$
S_{\mathrm{BH}}(k, D):=\hat{S}_{0} D^{\frac{D k}{2}} \Rightarrow\left\{\begin{array}{l}
R_{\mathrm{H}} \sim \ell_{\mathrm{P}} D^{\frac{k+1}{2}} \\
M_{\mathrm{H}} \sim M_{\mathrm{P}} D^{\frac{D k+1-k}{2}}
\end{array}\right.
$$

At large $D$, these families of black holes (i.e., these large- $D$ limits) exactly correspond to those studied by Emparan et al. [4] (and are related to the large- $D$ limits studied by Battacharya et al. [5]), where they fix $R_{\mathrm{H}}:=\hat{R}_{0} D^{\ell / 2}$ and study families of black holes with $\ell=1$ and $\ell=2$. (Note that their $D^{\ell / 2}$ is our $D^{(k+1) / 2}$.)

\section{A. Where should semiclassical gravity apply?}

We need clear criteria where the above semiclassical analysis applies. Clearly, we must have $R_{\mathrm{H}}>\ell_{\mathrm{P}}$ and $S_{\mathrm{BH}} \gg \mathcal{O}(1)$. However, there are further conditions.

Sub-Planckian curvature: First, we should require that the length scale defined by the curvature invariant $R_{\alpha \beta \mu \nu}^{2}:=\left.R_{\alpha \beta \mu \nu} R^{\alpha \beta \mu \nu}\right|_{r=R_{\mathrm{H}}}$ is sub-Planckian:

$$
\begin{aligned}
\left.\frac{R_{\alpha \beta \mu \nu}^{2}}{M_{\mathrm{P}}^{4}}\right|_{r=R_{\mathrm{H}}} & =\frac{(D-1)(D-2)^{2}(D-3)}{\left(R_{\mathrm{H}} / \ell_{\mathrm{P}}\right)^{4}} \\
& \simeq\left(\frac{\sqrt{D}}{S_{\mathrm{BH}}}\right)^{\frac{4}{D}} \ll 1 .
\end{aligned}
$$

This bound serves to ensure the subdominance of higher curvature corrections to the Einstein-Hilbert action under the assumption of technical naturalness.

Softness of radiation: Second, we fix the energy of the most likely quanta $E^{\star}$ to be lighter than the black hole:

$$
\frac{E^{\star}}{M_{\mathrm{H}}} \simeq \frac{D}{S_{\mathrm{BH}}} \ll 1
$$

The radiation cannot match a blackbody if $E^{\star} \sim M_{\mathrm{H}} \cdot{ }^{1}$

Quasistatic geometry: Third, we would like the black hole geometry to be relatively static during evaporation:

$$
\left|\frac{d R_{\mathrm{H}}}{d t}\right|=\left|\frac{d M_{\mathrm{H}}}{d t} \frac{d R_{\mathrm{H}}}{d M_{\mathrm{H}}}\right|=\frac{P_{\mathrm{BH}}}{D-3} \frac{R_{\mathrm{H}}}{M_{\mathrm{H}}} \ll 1 .
$$

Explicitly, as the Schwarzschild solution is static in Einstein gravity, if there is any appreciable departure from static geometry, characterized by $\left|d R_{\mathrm{H}} / d t\right| \ll 1$, then we

\footnotetext{
${ }^{1}$ We may also require $E^{\star}<M_{\mathrm{P}}$ [4]. If we insist on this constraint, then we find that $k \geq 3$. However, this does not seem directly connected to the integrity of semiclassicality. Amusingly, the shallow gravitational potential in $D \gg 1$ is less efficient at attenuating the energy in trans-Planckian Hawking quanta created near the horizon. In $D \gg 1$, trans-Planckian Hawking quanta may propagate to infinity, magnifying the trans-Planckian problem.
} 
assume that the system ceases to be semiclassical. ${ }^{2}$ This constraint is closely related to requiring $\dot{T} / T^{2} \ll 1$ for an approximately thermal emitter. However, since $\dot{T} / T^{2} \sim\left|\dot{R}_{\mathrm{H}}\right| / D$, this gives a considerably weaker constraint at large $D$.

Similarly, we may demand the black hole's decay width, given by its inverse lifetime, to be much smaller than its mass. However, this related constraint is much weaker than the constraint $\left|d R_{\mathrm{H}} / d t\right| \ll 1$.

Short scrambling times: In the next section, we show that there is a range of black holes that satisfy all of the above conditions, even though their scrambling times (12) are longer than their semiclassical half-life, $t_{\text {evap }} / 2$ (11). If true, this would imply that information would leak out of the black hole essentially unobscured. This is clearly incompatible with semiclassical Hawking radiation and forces us to impose the new condition

$$
t_{\text {scr }}<t_{\text {evap }}
$$

When $t_{\text {scr }}>t_{\text {evap }}$, unitary evolution of the black hole and its radiation field is in tension with this rapid decay.

\section{B. Bounds on $k$}

Here, we focus on which families of black holes defined in (13) can be consistent with the constraints (14)-(16). First, constraints (14) and (15) together imply

$$
\left.\begin{array}{l}
\frac{R_{\alpha \beta \mu \nu}^{2}}{M_{\mathrm{P}}^{4}} \sim D^{2-2 k} \ll 1 \\
\frac{E^{\star}}{M_{\mathrm{H}}} \sim D^{1-\frac{(D+1) k}{2}} \ll 1
\end{array}\right\} \Rightarrow k \geq 1 .
$$

If $k \in \mathbb{Z}_{<1}$, then both (a) median Hawking quanta have energies greater than the rest energy of the black hole and (b) the curvature scales are sub-Planckian. Alternatively, at large $D$, any black hole with $k \geq 1$ has sub-Planckian curvature and emits Hawking quanta whose energies are $\sim D^{1-(D+1) k / 2}$ smaller than its rest energy.

Due to the rapid evaporation times (which motivated this paper), one of the tightest constraints comes from requiring that the size/geometry of the black hole varies slowly over time, i.e., constraint (16). Relating $R_{\mathrm{H}} / M_{\mathrm{H}}$ to $S_{\mathrm{BH}}$ via Eq. (7), noting $P_{\mathrm{BH}}$ from Eq. (10), and writing $S_{\mathrm{BH}}=\hat{S}_{0}\left(\frac{D}{4 \pi}\right)^{\ell+(k D / 2)}$, we find

$$
\left|\frac{d R_{\mathrm{H}}}{d t}\right| \sim \frac{N_{D}}{D^{\ell}} \frac{(4 \pi)^{\ell}}{\hat{S}_{0}}\left(\frac{D^{1-k / 2}}{4 \pi}\right)^{D} \ll 1 \Rightarrow k \geq 2 .
$$

\footnotetext{
${ }^{2}$ One may argue that a quickly changing geometry is not per se in tension with semiclassicality since all corrections may still be captured in a self-consistent semiclassical expansion, in the spirit of [13]. However, as we will see, this plays no role at sufficiently large $D$ since quasistaticity does not pose the strongest bound on semiclassicality.
}

In pure gravity $N_{D}$ counts distinct graviton polarizations and grows quadratically: $N_{D}=\frac{D(D-3)}{2} \sim D^{2}$. Thus, the " $k=2$ " black holes evolve slowly at large $D$; they should be well described by semiclassical physics.

As a consequence of the above considerations, semiclassical black holes at large $D$ should have entropies that grow at least as quickly as $S_{\mathrm{BH}}>D^{D-2}$. However, it is straightforward to see that

$$
\left.\frac{t_{\text {evap }}}{t_{\text {scr }}}\right|_{D \gg 1}=\left\{\left(\frac{4 \pi}{D}\right)^{D} \frac{1}{N_{D}}\right\} \frac{S_{\mathrm{BH}}}{\log S_{\mathrm{BH}}} \frac{e^{2}}{2},
$$

where, for ease, we have used the simplified scalings in Eqs. (1) and (2) with properly restored order-one factors. Minimally, $N_{D}$ is at least $D(D-3) / 2 \simeq 8 \pi^{2}(D / 4 \pi)^{2}$. This lets us bound $t_{\text {evap }} / t_{\text {scr }}$ from above:

$$
\left.\frac{t_{\text {evap }}}{t_{\text {scr }}}\right|_{D \gg 1} \leq\left\{\left(\frac{4 \pi}{D}\right)^{D+2}\right\} \frac{S_{\mathrm{BH}}}{\log S_{\mathrm{BH}}} \frac{e^{2}}{(4 \pi)^{2}} .
$$

Thus, black holes with $\left(\frac{D}{4 \pi}\right)^{D-2}<S_{\mathrm{BH}}<\left(\frac{D}{4 \pi}\right)^{D+3} \log D$ evaporate parametrically faster than they scramble, without violating the bounds (14)-(16), derived from semiclassicality.

However, we recall that for evolution to be unitary, as happens within the semiclassical approximation, we must have $t_{\text {scr }} \ll t_{\text {evap. }}$. Thus, we need to impose

$$
S_{\mathrm{BH}}>\left(\frac{D}{4 \pi}\right)^{D+3} \log D .
$$

We observe an increase of the minimal entropy of semiclassical black holes by a factor of $D^{3} \log D$ as compared to the conventional bounds listed above. This is the main point of our paper.

This is also good news from a different perspective. Demanding $k \geq 2$ alone allows for black holes that have $t_{\text {evap }}<t_{\mathrm{P}}$, despite their Schwarzschild radius scaling as $R_{\mathrm{H}} \sim D^{3 / 2} \ell_{\mathrm{P}}$. Indeed, if $S_{\mathrm{BH}}:=\hat{S}_{0}(D / 4 \pi)^{D+l}$ for $-2<l<5 / 2$, then the evaporation times vanish in the strict large- $D$ limit:

$$
S_{\mathrm{BH}}:=\hat{S}_{0}\left(\frac{D}{4 \pi}\right)^{D+l} \Rightarrow \frac{t_{\mathrm{evap}}}{t_{\mathrm{P}}} \simeq \hat{S}_{0}\left(\frac{4 \pi}{D}\right)^{5 / 2-l} .
$$

The scrambling bound implies $l \geq 3$, such that these black holes cannot be semiclassical.

Let us end this section by noting that the effect appears less dramatic when expressed in terms of the Schwarzschild radius. The above condition becomes

$$
\frac{R_{\mathrm{H}}}{\ell_{\mathrm{P}}}>\left(\frac{4}{\Omega_{D-2}}\left(\frac{D}{4 \pi}\right)^{D+3} \log D\right)^{\frac{1}{D-2}} .
$$


For $D \gg 1$, this bounds $k \geq 2$, which in turn bounds the radii of semiclassical black holes by $R_{\mathrm{H}} / \ell_{\mathrm{P}} \gtrsim D^{3 / 2}$. An interesting consequence of this analysis is that semiclassical black hole temperatures are now bounded from above by $T_{\mathrm{H}} / M_{\mathrm{P}}=(D-3) \times\left(4 \pi R_{\mathrm{H}} / \ell_{\mathrm{P}}\right)^{-1}<(4 \pi \sqrt{D})^{-1}$.

\section{Light crossing and scrambling}

Equation (2) for the scrambling time is rather natural, irrespective of the number of dimensions. A black hole as a strongly coupled quantum system is characterized by its temperature $T_{\mathrm{H}}$ and an effective number of qubits $\sim S_{\mathrm{BH}}$. Fast scrambling is associated with an exponential growth of mixing, with a rate naturally set by the temperature. Complete scrambling then sets in when all qubits mix, after a time $t \sim T_{\mathrm{H}}^{-1} \log S_{\mathrm{BH}}$, in agreement with (2).

Nonetheless, one may reasonably wonder whether the physics of scrambling at large $D$ has hidden powers of $D$ in it which could close the window where $\left|d R_{\mathrm{H}} / d t\right| \ll 1$ and yet $t_{\text {evap }} / t_{\text {scr }} \ll 1$. While this would be extremely interesting, in particular, since it could shed important light on the dynamics behind scrambling even at small $D$, let us elaborate why we find it rather implausible.

Indeed, one might wonder if the physics at large $D$ could significantly alter the scrambling time such that $t_{\mathrm{scr}}$ is several factors of $D$ faster than the quoted result in (2). This is unlikely. The analysis that led to this estimate for the scrambling time is explicitly $D$ independent [11]. In particular, various plausibility arguments for the scaling of the scrambling time, such as the spreading of charge over the stretched horizon [10], suggest that if $t_{\text {scr }}$ at large- $D$ scales with an overall factor of $1 / D$ or faster, such spreading would be governed by a timescale that is either independent of the area of the horizon or inversely proportional to it. Neither seems likely.

Irrespectively of the precise dynamics, however, causality puts a stringent lower bound on the scrambling time. As we now show, even if scrambling were to happen at the fastest rate compatible with causal dynamics, our conclusions would remain essentially unchanged. To this end, we notice that however fast the scrambling dynamics may be, in Schwarzschild coordinates, a black hole cannot scramble information faster than the time it takes for light to travel between two points that are separated by twice its Schwarzschild radius, $2 R_{\mathrm{H}}$. We call this time the lightcrossing time and define it as $t_{\times}:=2 R_{\mathrm{H}}$. In these coordinates, this timescale represents the fastest possible propagation of information consistent with causality.

In the equations, we must have

$$
t_{\mathrm{scr}}\left(R_{\mathrm{H}}\right) \geq t_{\times}=2 R_{\mathrm{H}} .
$$

Now, the parametric $D$-scaling of $t_{\text {scr }}$ in (12) only weakly differs from $t_{\times}$:

$$
\frac{t_{\mathrm{scr}}}{t_{\times}}=\frac{\log S_{\mathrm{BH}}}{4 \pi R_{\mathrm{H}} T_{\mathrm{H}}}=\frac{\log S_{\mathrm{BH}}}{D-3} .
$$

Now, we focus on the interesting regime where $S_{\mathrm{BH}}=$ $\hat{S}_{0}(D / 4 \pi)^{D+\ell}$. We see that $t_{\text {evap }} / t_{\times}<1$ is still stronger than the two semiclassical constraints $\left|d R_{\mathrm{H}} / d t\right| \ll 1$ and $R_{\alpha \beta \gamma \delta}^{2} / M_{\mathrm{P}}^{4} \ll 1$. This follows straightforwardly from the fact that for these black holes, we have

$$
t_{\text {scr }} / t_{\times} \sim \log D
$$

and thus the constraints from Eqs. (20) and (21) translate into the constraint that

$$
\left.\frac{t_{\text {evap }}}{t_{\times}}\right|_{D \gg 1} \lesssim\left\{\left(\frac{4 \pi}{D}\right)^{D+2}\right\} \frac{S_{\mathrm{BH}}}{D} \frac{e^{2}}{(4 \pi)^{2}} .
$$

This more stringent constraint still leaves almost the entire window open. Explicitly, black holes with entropies within the range $\left(\frac{D}{4 \pi}\right)^{D-2}<S_{\mathrm{BH}}<\left(\frac{D}{4 \pi}\right)^{D+3}$ will evaporate parametrically faster than any causal signal could conceivably propagate in flat space. This holds even though (1) the geometry is static, i.e., $\left|d R_{\mathrm{H}} / d t\right| \ll 1$, and (2) the curvature at the horizon is sub-Planckian.

Because $t_{\times}$is the shortest, fastest way that a physical effect can possibly be consistent with causality, we see that the most general restrictions from fast scrambling come from requiring $t_{\text {evap }} / t_{\times}<1$. In particular, these constraints necessarily hold at large $D$.

Let us finally note that the above arguments rest on the assumption of quasistaticity, which, however, impeccably holds for $k \geq 2$. Since we are looking for those black holes with the largest $k$ that pose a challenge to a semiclassical analysis, we can safely assume the geometry to remain unchanged for the majority of the black hole's lifetime. In fact, were it not for the tension with scrambling, all black holes with $k \geq 2$ would, at sufficiently large $D$, be the perfect playground for semiclassics since backreaction on the geometry is essentially turned off. Only at the very latest stages of evaporation, when remaining lifetimes are much shorter than the light-crossing time, can corrections to quasistaticity become sizable. By then, however, most of the black hole's mass has been radiated away in a distinctly nonsemiclassical manner. We can illustrate this fact by comparing both $R_{\mathrm{H}}(t)$ and $S_{\mathrm{BH}}(t)$ as they easily follow from integrating Eq. (10) for $D=4$ and $D \gg 1$, as shown in Fig. 1.

\section{Short-lived black holes and string theory}

Clearly, when $D$ is $\mathcal{O}(1)$, the standard conditions for the curvature scale at the horizon, or the quasistatic nature of the black hole geometry, are, together, strong enough to dictate whether a black hole is well described by semiclassical physics. In other words, for $\mathcal{O}(1)$ values of $D$, scrambling times for such black holes are necessarily mисh shorter than their evaporation times. 

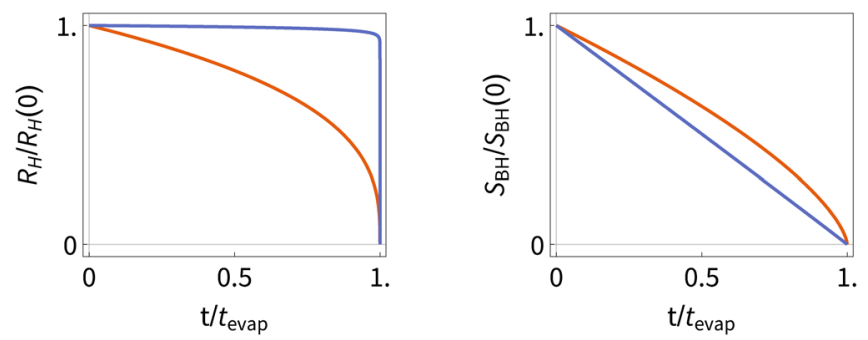

FIG. 1. Comparison of typical evaporation curves for $D=4$ (red) and $D \gg 1$ (blue), where for concreteness we picked $D=100$ for the latter. In the left panel, we plot $R_{\mathrm{H}}\left(t / t_{\text {evap }}\right)$, while on the right we display $S_{\mathrm{BH}}\left(t / t_{\text {evap }}\right)$.

Here, we briefly note that the lowest value of $D$ where $t_{\text {scr }} \sim t_{\text {evap }}$ for an otherwise "semiclassical" Schwarzschild black hole, i.e., one whose curvature scales are subPlanckian and whose geometry varies slowly in time (due to Hawking radiation), is significantly larger than $D=26$. In other words, the new condition for semiclassicality in this paper only applies above the upper critical dimension for consistent string theories.

\section{CONNECTION TO PREVIOUS WORK}

Previous work has also noted that black hole information, entropy, and evaporation can exhibit qualitatively new features at large $D$. Though recent work on the large- $D$ limit of general relativity has focused on classical features of the theory, they are explicitly aware of the high luminosity (and short timescales) that would be associated with semiclassical, and fully quantum, gravity in large $D$.

Moreover, semiclassical aspects of black hole physics at large $D$ have been a direct focus of [6-9]. In particular, the Hawking luminosity was explicitly found in [6], and Refs. [7-9] have discussed the relative size of black hole entropy as compared to the entropies of unbound systems of weakly gravitating matter at large $D$.

In particular, Ref. [8] prominently discusses possible tension between basic principles of black hole entropy and properties of weakly gravitating systems when the entropy of a black hole is $S_{\mathrm{BH}}(D) \lesssim(D / 4 \pi)^{D+\ell}$ if $\ell$ lies in the interval $\ell \in\left(\frac{1}{2}, 1\right)$. In our present context, it is very amusing to note that these black holes fall squarely within the family of black holes for which the scrambling bound is the strongest. In fact, our inequality (22) resolves possible tension by demonstrating that a semiclassical analysis of such black holes should not be trusted.

Our results are similar in spirit to results derived in the context of a large number $N$ of gravitationally interacting species [14]. Their presence opens up the phase space in a similar fashion as going to large $D$. Consequently, evaporation times and scrambling times can become comparable for sufficiently large $N$, which again bounds the validity of the semiclassical approximation [15]. Interestingly, in the case of species, breakdown of semiclassicality is directly tied to a loss of perturbative unitarity $[14,16]$. We leave the question of exploring such a connection in the context of large- $D$ physics $[17,18]$ for the future.

\section{CONCLUSIONS AND FUTURE WORK}

Analyzing the first quantum corrections to black holes in a large number of dimensions, $D \gg 1$, reveals rather curious properties. The presence of this large dimensionless number has the capacity to significantly alter the naive intuition, gleaned from black holes in four and five dimensions, for the timescales in unitary evaporation.

Indeed, in $D \gg 1$, black hole temperatures grow linearly with $D$. Explicitly, $T_{\mathrm{H}} R_{\mathrm{H}} \sim D$, which comes from the gradient of $g_{00}(r) \sim 1-\left(\frac{R_{\mathrm{H}}}{r}\right)^{D}$ at the horizon. Further, the Hawking luminosity grows factorially with $D$. This growth is mainly due to the growth of the available phase space with $D$. Neither of these scalings is surprising. Yet, they inexorably lead to very short evaporation timescales for even relatively massive black holes, with $\frac{M_{\mathrm{H}}}{M_{\mathrm{P}}} \lesssim\left(\frac{D}{4 \pi}\right)^{D}$, which may lead to new observations.

Within this paper, we have taken the conservative approach of interpreting all possible sources of tension that appear within the semiclassical approximation as pointing to the latter's demise. In particular, we have identified a range of parameters in which otherwise semiclassical black holes appear to evaporate much faster than they scramble information. Since this is incompatible with everything we understand about the microscopic dynamics of scrambling, we have taken this as a signal that in a large number of dimensions, semiclassical physics breaks down at scales significantly larger than naively expected.

Interestingly, this realization helps us to clarify nontrivial puzzles that have arisen in the literature, such as the possibility of having hyperentropic matter at sufficiently large $D$ [7] that violates the Bekenstein bound [19]. ${ }^{3}$ While recent arguments argue against the validity of such conclusions on different grounds [9], we think that our picture provides a complementary and conceptually very simple explanation for why such apparent tension arises outside the regime of validity of semiclassical gravity.

One of the main assumptions underlying this work is the validity of expression (12) for the scrambling time in any $D$. From a certain point of view, this may seem unnatural. After all, it implies that the entire $D$ dependence of the scrambling time lies only in the $D$ dependence of temperature and entropy. One may thus wonder whether corrections to this expression, relevant only at sufficiently large $D$, could lead to a decrease of thermalization

\footnotetext{
${ }^{3}$ Such matter does not violate the covariant entropy bound, which is a factor of $\frac{2}{D-2}$ weaker in $D \neq 4$ [20]. It would be interesting to see whether the fact that the Bekenstein bound is potentially violated only outside the semiclassical regime can shed light on the mismatch of the bounds.
} 
timescales such that $t_{\text {scr }}<t_{\text {evap }}$ for any $D$ and $S_{\mathrm{BH}}>1$. On the other hand, the expected nonlocal nature of scrambling dynamics [11] appears to directly imply scrambling to be insensitive to the number of spacetime dimensions. We hope to shed more light on this issue in future work. We close with a few comments.

First, although holographic dualities between conformal field theories (CFTs) in $D$ dimensions and gravitational theories in $(D+1)$ dimensions are not expected to be "nice" (or, perhaps, to exist at all) when $D \gg 1$, there are hints that CFTs might be very simple at large $D$ [21]. It might be interesting to see if this is relevant in small black hole evaporation in $(D+1)$ dimensions.

Second, in [22] the near-horizon geometries of black holes at large $D$ were found to match those in the specific $2 D$ dilaton gravity model of Callan, Giddings, Harvey and Strominger (CGHS) [23]. Strikingly, aspects of this analogy seem to persist at the semiclassical level. Explicitly, black holes at large $D$ and in the $2 D$ CGHS model have mass-independent temperatures. As this and other semiclassical aspects of large- $D$ BHs are captured by dilaton gravity in $2 D$, this may suggest that corresponding features of the information problem also be captured by the much simpler aspects of gravity in two dimensions. It would be extremely interesting to study whether this bridge between $2 D$ and large $D$ could be made more robust and, if so, whether it offered new insights on the information problem in $D$ dimensions.

Finally, similar considerations to the ones presented here have played an important role in formulating gravitational consistency conjectures, such as the weak gravity [24] and swampland [25] conjectures. It will be interesting to see whether large- $D$ physics can yield nontrivial insights there, like in the case of cosmic censorship [26,27].

\section{ACKNOWLEDGMENTS}

We thank Peter Denton, Roberto Emparan, Troels Harmark, Cindy Keeler, Niels Obers, and Marta Orselli for stimulating discussions and important comments on the manuscript, as well as Gia Dvali and Oriol Pujolas for useful discussions and Nima Arkani-Hamed for emphasizing the fundamental importance of $t_{\mathrm{scr}}<t_{\text {evap }}$. D. A. M. is supported by a Nordita fellowship under the grant "Exact Results in Gauge and String Theories" from the Knut and Alice Wallenberg Foundation. The work of N.W. was supported by FNU Grant No. DFF-6108-00340.

\section{APPENDIX: $K_{D}$ AND $L_{D}$}

The function $K_{D}$ introduced in Eq. (10), for black hole lifetimes, is explicitly

$$
K_{D}:=2 e^{2} \zeta(D)\left(\frac{D-1}{D^{2}}\right)\left(\frac{b_{\text {crit }}}{R_{\mathrm{H}}}\right)^{D-2}\left(\frac{D-3}{D}\right)^{D}
$$

and $L_{D}$ in Eq. (11), also for black hole lifetimes, is

$$
L_{D}:=\left(\frac{D-3}{D-1} \frac{D-2}{D}\right) \sqrt{\frac{e}{2}} \sqrt{\frac{4 \pi}{D}}\left(\frac{4}{\Omega_{D-2}}\right)^{\frac{1}{D-2}} .
$$

Note that $L_{D}, K_{D} \rightarrow 1$ as $D \rightarrow \infty$. While we do not give an expression for $\gamma_{D}\left(R_{\mathrm{H}}\right)$, we recall that $\lim _{D \rightarrow \infty} \gamma_{D}\left(R_{\mathrm{H}}\right)=1[6]$.
[1] S. W. Hawking, Phys. Rev. D 14, 2460 (1976).

[2] D. N. Page, Phys. Rev. Lett. 71, 1291 (1993).

[3] D. N. Page, J. Cosmol. Astropart. Phys. 09 (2013) 028.

[4] R. Emparan, R. Suzuki, and K. Tanabe, J. High Energy Phys. 06 (2013) 009.

[5] S. Bhattacharyya, A. De, S. Minwalla, R. Mohan, and A. Saha, J. High Energy Phys. 04 (2016) 076.

[6] S. Hod, Classical Quantum Gravity 28, 105016 (2011).

[7] S. Hod, Phys. Lett. B 695, 294 (2011).

[8] S. Hod, Phys. Lett. B 700, 75 (2011).

[9] S. Hod, Phys. Rev. D 97, 126012 (2018).

[10] P. Hayden and J. Preskill, J. High Energy Phys. 09 (2007) 120.

[11] Y. Sekino and L. Susskind, J. High Energy Phys. 10 (2008) 065.

[12] R. Emparan, G. T. Horowitz, and R. C. Myers, Phys. Rev. Lett. 85, 499 (2000).

[13] C.-I. Kuo and L. Ford, Phys. Rev. D 47, 4510 (1993).

[14] G. Dvali, Fortschr. Phys. 58, 528 (2010).
[15] G. Dvali and C. Gomez, Phys. Lett. B 674, 303 (2009).

[16] G. Dvali and M. Redi, Phys. Rev. D 77, 045027 (2008).

[17] A. Strominger, Phys. Rev. D 24, 3082 (1981).

[18] N. E. J. Bjerrum-Bohr, Nucl. Phys. B684, 209 (2004).

[19] J. D. Bekenstein, Phys. Rev. D 23, 287 (1981).

[20] R. Bousso, J. High Energy Phys. 04 (2001) 035.

[21] A. L. Fitzpatrick, J. Kaplan, and D. Poland, J. High Energy Phys. 08 (2013) 107.

[22] R. Emparan, D. Grumiller, and K. Tanabe, Phys. Rev. Lett. 110, 251102 (2013).

[23] C. G. Callan, Jr., S. B. Giddings, J. A. Harvey, and A. Strominger, Phys. Rev. D 45, R1005 (1992).

[24] N. Arkani-Hamed, L. Motl, A. Nicolis, and C. Vafa, J. High Energy Phys. 06 (2007) 060.

[25] C. Vafa, arXiv:hep-th/0509212.

[26] R. Penrose, Riv. Nuovo Cimento 1, 252 (1969) [Gen. Relativ. Gravit. 34, 1141 (2002)].

[27] T. Andrade, R. Emparan, D. Licht, and R. Luna, J. High Energy Phys. 09 (2019) 099. 